TRANSACTIONS OF THE

AMERICAN MATHEMATICAL SOCIETY

Volume 363, Number 12, December 2011, Pages 6337-6349

S 0002-9947(2011)05359-6

Article electronically published on July 20, 2011

\title{
GLOBAL HYPOELLIPTICITY, GLOBAL SOLVABILITY AND NORMAL FORM FOR A CLASS OF REAL VECTOR FIELDS ON A TORUS AND APPLICATION
}

\author{
G. PETRONILHO
}

\begin{abstract}
The main purpose of this paper is to present a class of real vector fields defined on a torus for which the concepts of global hypoellipticity and global smooth solvability are equivalent. Furthermore, such a vector field is globally hypoelliptic if and only if its adjoint is globally hypoelliptic, and therefore we can reduce it to its normal form. As an application, we study global $C^{\infty}$ solvability for certain classes of sub-Laplacians.
\end{abstract}

\section{INTRODUCTION}

In this paper we will analyze the following questions about a real vector field $L=\sum_{j=1}^{N} a_{j}(x) \partial_{x_{j}}$, where $a_{j} \in C^{\infty}\left(\mathbb{T}^{N}\right), j=1, \ldots, N$, which does not vanish on $\mathbb{T}^{N}$ :

Q1: Is it true that $L$ is globally hypoelliptic in $\mathbb{T}^{N}$ iff its adjoint is globally hypoelliptic in $\mathbb{T}^{N}$ ?

Concerning this question we recall that if its adjoint $L^{*}$ is globally hypoelliptic in $\mathbb{T}^{N}$, then it follows from Theorem 2.2 in Chen and Chi $[\mathrm{CC}$ that $L$ is globally hypoelliptic in $\mathbb{T}^{N}$. We do not know if the converse of Theorem 2.2 in [CC] is true. However, Himonas, Petronilho and dos Santos [HPS] have proved it when $N=2$.

Q2: Is it true that $L$ is globally $C^{\infty}$ solvable in $\mathbb{T}^{N}$ iff either its adjoint, $L^{*}$, or $L$ is globally hypoelliptic in $\mathbb{T}^{N}$ ?

Q3: Is it true that $L$ is globally $C^{\infty}$ solvable in $\mathbb{T}^{N}$ iff $L^{*}$ is globally $C^{\infty}$ solvable in $\mathbb{T}^{N}$ ?

Concerning these questions it is well known that a standard functional analysis argument applies: the global hypoellipticity of $L^{*}$ implies the global solvability of $L$. Since global hypoellipticity of $L^{*}$ implies global hypoellipticity of $L$, as we have commented above, it follows that $L$ is globally $C^{\infty}$ solvable in $\mathbb{T}^{N}$. Also, it is well known that global solvability, even global $C^{\infty}$ solvability, of $L$ in $\mathbb{T}^{N}$, in general, does not imply that either $L^{*}$ or $L$ is globally hypoelliptic in $\mathbb{T}^{N}$. For instance, consider

$$
L=\partial_{t}+\lambda \partial_{x}
$$

Received by the editors October 20, 2009.

2010 Mathematics Subject Classification. Primary 35A01, 35F05, 35H10.

Key words and phrases. Global hypoellipticity, global solvability, normal form.

The author was partially supported by CNPq and Fapesp.

(C)2011 American Mathematical Society Reverts to public domain 28 years from publication 6337 
with $(t, x) \in \mathbb{T}^{2}$ and $\lambda$ is a rational number. The vector field $L$ is globally $C^{\infty}$ solvable in $\mathbb{T}^{2}$ but $L^{*}(=-L)$ and $L$ are not globally hypoelliptic in $\mathbb{T}^{2}$.

We would also like to point out that $\mathbf{Q 1}$ is related to the following question:

Q4: If $L$ is globally hypoelliptic in $\mathbb{T}^{N}$, is there a diffeomorphism of $\mathbb{T}^{N}$ onto $\mathbb{T}^{N}$ that takes $L$ into

$$
\sum_{j=1}^{N} A_{j} \partial_{y_{j}}
$$

where the real numbers $A_{j}$ satisfy some Diophantine condition?

We now recall some well-known results about question $\mathbf{Q 4}$.

Theorem 1.1 (see Hounie $\mathrm{Hou}$ ). Let $X$ be a real vector field on $\mathbb{T}^{2}$ and suppose that $X$ does not vanish on $\mathbb{T}^{2}$. If $X$ is a globally hypoelliptic vector field on $\mathbb{T}^{2}$, then there exists a diffeomorphism of $\mathbb{T}^{2}$ onto $\mathbb{T}^{2}$ that takes $X$ into

$$
f(s, t)\left(\partial_{s}+A \partial_{t}\right),
$$

where the constant $A$ is an irrational non-Liouville number and $f \in C^{\infty}\left(\mathbb{T}^{2}\right)$ is a non-vanishing function.

In $\mathbb{T}^{n}$ there exists a new reduction theorem for real vector fields due to Chen and $\mathrm{Chi} \mathrm{CC}$ :

Theorem 1.2. Let $X$ be a real vector field on $\mathbb{T}^{N}$. Then the transpose of $X$ is a globally hypoelliptic operator on $\mathbb{T}^{N}$ if and only if there exist coordinates y on $\mathbb{T}^{N}$ in which $X$ admits the form

$$
X=\sum_{j=1}^{N} A_{j} \partial_{y_{j}}
$$

with the real numbers $A_{1}, \cdots, A_{N}$ satisfying the following Diophantine condition: there exist positive constants $C$ and $K$ such that

$$
\left|\sum_{j=1}^{N} \xi_{j} A_{j}\right| \geq \frac{C}{(1+|\xi|)^{K}}, \forall \xi \in \mathbb{Z}^{N} \backslash\{0\} .
$$

Remark 1.3. Theorem 1.2 gives new results on normal forms of real vector fields on $\mathbb{T}^{N}$ even for $N=2$ (cf. Theorem 1.4 in Greenfield and Wallach GW2]).

We notice that if $L$ is globally hypoelliptic in $\mathbb{T}^{N}$ and $\mathbf{Q} \mathbf{1}$ has a positive answer, then it follows from Theorem 1.2 that $\mathbf{Q} 4$ has a positive answer as well.

The main goal here is to present a special class of real vector fields defined in a torus such that the concepts of global hypoellipticity, global $C^{\infty}$ solvability and reduction to a normal form are all equivalent (see Theorem 3.1).

As an application we consider the following class of sub-Laplacians defined on the torus $\mathbb{T}^{n+m}$ by $P=-\Delta_{t}-L_{x}^{2}$, where $t \in \mathbb{T}^{n}, x \in \mathbb{T}^{m}, L=\sum_{j=1}^{m} a_{j}(x) \partial_{x_{j}}$ and we prove, under some conditions, that $P$ is globally $C^{\infty}$ solvable in $\mathbb{T}^{n+m}$ if and only if $L$ is globally $C^{\infty}$ solvable in $\mathbb{T}^{m}$ (see Theorem 4.3) . As alluded to above, in order to study global solvability of an operator, it is natural to study global hypoellipticity for its adjoint. Thus, we start by analyzing the global hypoellipticity for the operator $P^{*}$. When $L$ is a real vector field that does not vanish on $\mathbb{T}^{m}$ we prove that $P^{*}$ is globally hypoelliptic in $\mathbb{T}^{n+m}$ iff $L^{*}$ is globally hypoelliptic in $\mathbb{T}^{m}$, but when the kernel of $L^{*}$ is spanned by a single smooth and non-vanishing 
function, then we show that $P$ is globally hypoelliptic in $\mathbb{T}^{n+m}$ iff $L$ is globally hypoelliptic in $\mathbb{T}^{m}$ (see Theorem 4.1). Theorem 4.1 generalizes part of Theorem 6.2 in [HPS. Finally we present an example where the hypotheses of our Theorem 4.3 about global $C^{\infty}$ solvability of operator $P$ are fulfilled.

For more results on the problem of global hypoellipticity we refer the reader to the works by Amano [A], Fujiwara and Omori [FO, Greenfield and Wallach [GW1, Himonas [Hi], Himonas and Petronilho HP1], HP2] and Omori and Kobayashi $\mathrm{OK}$, and the references therein.

For results on interesting open problems of local and global solvability we refer the reader to the following papers as well as the references therein: Albanese and Zanghirati [AZ], Bergamasco, Cordaro and Petronilho [BCP], Gramchev, Popivanov and Yoshino GPY1 and GPY2, Petronilho [P1] and [P2].

For results on diffeomorphisms that are globally conjugated to a rotation we refer the reader to Brjuno $[\mathrm{Br}]$, Herman $[\mathrm{He}$, Yoccoz $[\mathrm{Y}]$ and the references therein.

This paper is structured as follows. In section 2 we provide some definitions in order to, in section 3, state our result about global hypoellipticity, global solvability and reduction to the normal form for certain class of real vector fields. In section 4 we apply the results obtained in the previous section in order to study global solvability and global hypoellipticity for certain classes of sub-Laplacians.

\section{Definitions}

Let $P$ be a linear partial differential operator with coefficients in $C^{\infty}\left(\mathbb{T}^{N}\right)$.

Definition 2.1. We say that

$$
P: C^{\infty}\left(\mathbb{T}^{N}\right) \rightarrow C^{\infty}\left(\mathbb{T}^{N}\right)
$$

is globally $C^{\infty}$ solvable in $\mathbb{T}^{N}$ if for every

$$
f \in\left\{h \in C^{\infty}\left(\mathbb{T}^{N}\right):\langle w, h\rangle=0, \forall w \in \operatorname{Ker} P^{*}\right\}
$$

there exists $u \in C^{\infty}\left(\mathbb{T}^{N}\right)$ such that $P u=f$, where

$$
P^{*}: D^{\prime}\left(\mathbb{T}^{N}\right) \rightarrow D^{\prime}\left(\mathbb{T}^{N}\right)
$$

is the transpose of $P$.

Definition 2.2. We say that

$$
P: D^{\prime}\left(\mathbb{T}^{N}\right) \rightarrow D^{\prime}\left(\mathbb{T}^{N}\right)
$$

is globally solvable in $\mathbb{T}^{N}$ if for every

$$
f \in\left\{h \in C^{\infty}\left(\mathbb{T}^{N}\right):\langle w, h\rangle=0, \forall w \in \operatorname{Ker} P^{*}\right\}
$$

there exists $u \in D^{\prime}\left(\mathbb{T}^{N}\right)$ such that $P u=f$, where

$$
P^{*}: C^{\infty}\left(\mathbb{T}^{N}\right) \rightarrow C^{\infty}\left(\mathbb{T}^{N}\right)
$$

is the transpose of $P$.

Definition 2.3. We say that

$$
P: D^{\prime}\left(\mathbb{T}^{N}\right) \rightarrow D^{\prime}\left(\mathbb{T}^{N}\right)
$$

is globally hypoelliptic in $\mathbb{T}^{N}$ if the conditions $u \in D^{\prime}\left(\mathbb{T}^{N}\right)$ and $P u \in C^{\infty}\left(\mathbb{T}^{N}\right)$ imply that $u \in C^{\infty}\left(\mathbb{T}^{N}\right)$. 
Remark 2.4. It is easily seen that if $P$ is globally solvable in $\mathbb{T}^{N}$ and globally hypoelliptic in $\mathbb{T}^{N}$, then $P$ is globally $C^{\infty}$ solvable in $\mathbb{T}^{N}$.

\section{REsults}

In the next result we present a class of vector fields for which $\mathbf{Q 1}, \mathbf{Q} 2$ and Q3 hold true for any dimension and we can write these vector fields in their normal form.

Theorem 3.1. Let $L=\sum_{j=1}^{N} a_{j}(x) \partial_{x_{j}}$ be a smooth real vector field on $\mathbb{T}^{N}$. Assume that Ker $L^{*}=[w]$, where $w \in C^{\infty}\left(\mathbb{T}^{N}\right)$ and $w(x) \neq 0, \forall x \in \mathbb{T}^{N}$. Then the following conditions are equivalent:

(1) There exists a diffeomorphism $\tau: \mathbb{T}^{N} \rightarrow \mathbb{T}^{N}, y=\tau(x)$ such that

$$
L=\sum_{j=1}^{N} A_{j} \partial_{y_{j}}
$$

with the numbers $A_{1}, \cdots, A_{N}$ satisfying the condition: there exist $K>$ $0, C>0$ such that

$$
\left|\sum_{j=1}^{N} \xi_{j} A_{j}\right| \geq \frac{C}{(1+|\xi|)^{K}}, \quad \forall\left(\xi_{1}, \cdots, \xi_{N}\right) \in \mathbb{Z}^{N} \backslash\{0\} .
$$

(2) $L^{*}$ is globally hypoelliptic in $\mathbb{T}^{N}$.

(3) $L$ is globally hypoelliptic in $\mathbb{T}^{N}$.

(4) $L$ is globally $C^{\infty}$ solvable in $\mathbb{T}^{N}$.

(5) $L^{*}$ is globally $C^{\infty}$ solvable in $\mathbb{T}^{N}$.

Remark 3.2. Before starting the proof we would like to point out that for the class of vector fields studied in Theorem 3.1, global $C^{\infty}$ solvability implies global solvability.

Remark 3.3. We recall that if $L^{*}$ is globally hypoelliptic in $\mathbb{T}^{N}$, then $\operatorname{Ker} L^{*}=[w]$, where $w \in C^{\infty}\left(\mathbb{T}^{N}\right)$ and $w(x) \neq 0, \forall x \in \mathbb{T}^{N}$; see Theorem 2.1 in [CC]. On the other hand, there exist real vector fields such that their adjoints are not globally hypoelliptic in $\mathbb{T}^{N}$ but that their kernel is spanned by just one function $w$ as described above. For instance, consider in $\mathbb{T}^{2}$ the vector field

$$
L=\partial_{t}+\lambda \partial_{x}
$$

where $\lambda$ is a Liouville number. It is well known that $L^{*}=-L$ is not globally hypoelliptic in $\mathbb{T}^{2}$ but

$$
\operatorname{Ker} L^{*}=\operatorname{Ker} L=[1] \text {. }
$$

Proof. It follows from $[\mathrm{CC}$ that $(1) \Leftrightarrow(2),(2) \Rightarrow(3)$ and $(2) \Rightarrow(4)$. $(3) \Rightarrow(2)$ : Let $u \in D^{\prime}\left(\mathbb{T}^{N}\right)$ be such that

$$
L^{*} u=f \in C^{\infty}\left(\mathbb{T}^{N}\right) .
$$


We now notice that for any $v \in D^{\prime}\left(\mathbb{T}^{N}\right)$ we have

$$
\begin{aligned}
L^{*}(w v) & =-L(w v)-\left(\sum_{j=1}^{N} \frac{\partial a_{j}}{\partial x_{j}}\right)(w v) \\
& =-(L w) v-w L v-\left(\sum_{j=1}^{N} \frac{\partial a_{j}}{\partial x_{j}}\right)(w v) \\
& =\left(L^{*} w\right) v-w L v=-w L v
\end{aligned}
$$

since $L^{*} w=0$.

It follows from (3.2) and (3.3) that

$$
f=L^{*} u=L^{*}\left(w \frac{1}{w} u\right)=-w L\left(\frac{1}{w} u\right) .
$$

Therefore

$$
L\left(\frac{1}{w} u\right)=-\frac{1}{w} f \in C^{\infty}\left(\mathbb{T}^{N}\right) .
$$

By using the hypotheses that $L$ is globally hypoelliptic in $\mathbb{T}^{N}$, it follows from (3.4) that

$$
\frac{1}{w} u=g \in C^{\infty}\left(\mathbb{T}^{N}\right)
$$

in turn implies that $u \in C^{\infty}\left(\mathbb{T}^{N}\right)$. Hence $L^{*}$ is globally hypoelliptic in $\mathbb{T}^{N}$.

$(4) \Rightarrow(3):$ Let $u \in D^{\prime}\left(\mathbb{T}^{N}\right)$ be such that

$$
L u=f \in C^{\infty}\left(\mathbb{T}^{N}\right) .
$$

Let $h \in \operatorname{Ker} L^{*}$. Then $h=c w$ with $c$ being a constant since $\operatorname{Ker} L^{*}=[w]$. Thus,

$$
\int_{\mathbb{T}^{N}} f h=\langle f, h\rangle=c\langle f, w\rangle=c\langle L u, w\rangle=c\left\langle u, L^{*} w\right\rangle=c\langle u, 0\rangle=0 .
$$

Thanks to this fact and thanks to the hypothesis that $L$ is globally $C^{\infty}$ solvable in $\mathbb{T}^{N}$, there exists $v \in C^{\infty}\left(\mathbb{T}^{N}\right)$ such that $L v=f$. Therefore we can conclude that $L u=L v$. Hence,

$$
L(u-v)=0 .
$$

It follows from this and from (3.3) that

$$
L^{*}[w(u-v)]=-w L(u-v)=0 .
$$

Again, thanks to the fact that Ker $L^{*}=[w]$ it follows from $L^{*}[w(u-v)]=0$ that

$$
u-v=c=\text { constant }
$$

in turn implies that $u \in C^{\infty}\left(\mathbb{T}^{N}\right)$ and therefore $L$ is globally hypoelliptic in $\mathbb{T}^{N}$.

$(3) \Rightarrow(5)$ : Since $(3) \Rightarrow(2)$ and also (3) implies that $L^{*}$ is globally solvable in $\mathbb{T}^{N}$ it follows from Remark 2.4 that $L^{*}$ is globally $C^{\infty}$ solvable in $\mathbb{T}^{N}$.

$(5) \Rightarrow(2):$ Let $u \in D^{\prime}\left(\mathbb{T}^{N}\right)$ be such that

$$
L^{*} u=f \in C^{\infty}\left(\mathbb{T}^{N}\right) .
$$

For $T \in \operatorname{Ker} L$ we have $\langle T, f\rangle=\left\langle T, L^{*} u\right\rangle=\langle L T, u\rangle=0$. Thus it follows from the hypothesis that there exists $v \in C^{\infty}\left(\mathbb{T}^{N}\right)$ such that

$$
L^{*} v=f
$$


since $L^{*}$ is globally $C^{\infty}$ solvable. It follows from (3.6) and (3.7) that $u-v \in$ Ker $L^{*}=[w]$ and therefore $u \in C^{\infty}\left(\mathbb{T}^{N}\right)$.

Remark 3.4. The hypothesis that Ker $L^{*}=[w]$, where $w \in C^{\infty}\left(\mathbb{T}^{N}\right)$ and $w(x) \neq$ $0, \forall x \in \mathbb{T}^{N}$, is superfluous for the proofs: $(1) \Leftrightarrow(2),(2) \Rightarrow(3)$ and $(2) \Rightarrow(4)$, while we need it for the proofs $(4) \Rightarrow(3)$ and $(5) \Rightarrow(2)$. For the proofs $(3) \Rightarrow(2)$ and $(3) \Rightarrow(5)$ it suffices the existence of the function $w \in C^{\infty}\left(\mathbb{T}^{N}\right)$ and $w(x) \neq$ $0, \forall x \in \mathbb{T}^{N}$ that $L^{*} w=0$.

Remark 3.5. It follows from (3.3) that Ker $L=\{$ constants $\}$, provided Ker $L^{*}=[w]$, where $w \in C^{\infty}\left(\mathbb{T}^{N}\right)$ with $w(x) \neq 0, \forall x \in \mathbb{T}^{N}$.

We now present a sufficient condition in order to guarantee that the existence of a function $w \in C^{\infty}\left(\mathbb{T}^{N}\right)$ such that $L^{*} w=0$ with $w(x) \neq 0, \forall x \in \mathbb{T}^{N}$ implies that Ker $L^{*}=[w]$; more precisely,

Lemma 3.6. If $L$ is globally hypoelliptic in $\mathbb{T}^{N}$ and there exists a function $w \in$ $C^{\infty}\left(\mathbb{T}^{N}\right)$ such that $L^{*} w=0$ with $w(x) \neq 0, \forall x \in \mathbb{T}^{N}$, then $\operatorname{Ker} L^{*}=[w]$.

Proof. Let $T \in D^{\prime}\left(\mathbb{T}^{N}\right)$ be such that $L^{*} T=0$. We set $g=\frac{1}{w} T \in D^{\prime}\left(\mathbb{T}^{N}\right)$. Thus, we have $T=w g$ and it follows from (3.3) that

$$
0=L^{*} T=L^{*}(w g)=-w(L g) .
$$

Hence, $L g=0$. Since $L$ is globally hypoelliptic in $\mathbb{T}^{N}$ we obtain $g \in C^{\infty}\left(\mathbb{T}^{N}\right)$ and therefore $T=w g \in C^{\infty}\left(\mathbb{T}^{N}\right)$.

Since $L$ is a vector field we have $L\left(g^{k}\right)=0, \forall k \in\{0,1,2, \ldots\}$. Therefore Ker $L \supset\left\{1, g, g^{2}, \ldots, g^{j}, \ldots\right\}$.

Now an application of the Rellich lemma implies that $\operatorname{dim} \operatorname{Ker} L<\infty$, since $L$ is globally hypoelliptic in $\mathbb{T}^{N}$. Thus $1, g, g^{2}, \ldots, g^{k}$ are linearly dependent for $k$ sufficiently large. This implies

$$
\sum_{j=0}^{k} a_{j} g^{j}=0
$$

with $a_{j} \in \mathbb{C}$ and not all $a_{j}$ zero. Hence $g$ can take at most $k$ different values. Since $\mathbb{T}^{N}$ is connected, $g$ is constant. Thus we can conclude that $T=c w$ with $c$ a constant and therefore $T \in[w]$.

On the other hand, if $T=c w$ with $c$ a constant, it is easily seen that $L^{*} T=0$.

\section{Application}

We start this section by recalling that in [HPS] the authors consider the operator $P=-\partial_{t}^{2}-\left(\partial_{x_{1}}+a\left(t, x_{1}, x_{2}\right) \partial_{x_{2}}\right)^{2}$ and under the hypothesis that all points in $\mathbb{T}^{3}$ are of infinite type for the vector fields $X=-\partial_{t}$ and $L=\partial_{x_{1}}+a\left(t, x_{1}, x_{2}\right) \partial_{x_{2}}$ they have proved that $P$ is globally hypoelliptic in $\mathbb{T}^{3}$ iff the vector field $L=$ $\partial_{x_{1}}+a\left(x_{1}, x_{2}\right) \partial_{x_{2}}$ is globally hypoelliptic in $\mathbb{T}^{2}$. Note that the infinite type condition implies that the coefficient $a$ depends only on the variables $x_{1}$ and $x_{2}$ and their operator can be written as $P=-\partial_{t}^{2}-\left(\partial_{x_{1}}+a\left(x_{1}, x_{2}\right) \partial_{x_{2}}\right)^{2}$. Thus the study of the global hypoellipticity for $P$ is equivalent to the study of the global hypoellipticity for the vector field $L$.

Motivated by this situation we will analyze global $C^{\infty}$ solvability and global hypoellipticity for the following class of sub-Laplacians:

$$
P=-\Delta_{t}-L_{x}^{2}
$$


where $t \in \mathbb{T}^{n}$ and $L_{x}=\sum_{j=1}^{m} a_{j}(x) \partial_{x_{j}}$ does not vanish on $\mathbb{T}^{m}$ and $a_{j} \in C^{\infty}\left(\mathbb{T}^{m} ; \mathbb{R}\right)$, $j=1, \ldots, m$. We will use the notation

$$
P=-\Delta-L^{2}
$$

It is well known that a linear operator is globally solvable in $\mathbb{T}^{N}$ provided its adjoint is globally hypoelliptic in $\mathbb{T}^{N}$. Thus, in order to study global $C^{\infty}$ solvability for our operator $P$, given by (4.1), we start by studying the global hypoellipticity for $P^{*}$. More precisely, we prove the following.

Theorem 4.1. Let $P$ be given by (4.1). Then, $\mathbb{T}^{m}$.

1) $P^{*}$ is globally hypoelliptic in $\mathbb{T}^{n+m}$ if and only if $L^{*}$ is globally hypoelliptic in

2) If we assume that $\operatorname{Ker} L^{*}=[w]$, where $w$ is as in Theorem 3.1, then $P$ is globally hypoelliptic in $\mathbb{T}^{n+m}$ if and only if $L$ is globally hypoelliptic in $\mathbb{T}^{m}$.

Proof of 1). Necessity: Assume that $L^{*}$ is not globally hypoelliptic in $\mathbb{T}^{m}$. Thus, there exists $u \in D^{\prime}\left(\mathbb{T}^{m}\right) \backslash C^{\infty}\left(\mathbb{T}^{m}\right)$ such that $L^{*} u=f \in C^{\infty}\left(\mathbb{T}^{m}\right)$ and therefore we have

$$
\left(L^{*}\right)^{2} u=L^{*}\left(L^{*} u\right)=L^{*} f=g \in C^{\infty}\left(\mathbb{T}^{m}\right) .
$$

Since we have $\left(L^{2}\right)^{*}=\left(L^{*}\right)^{2}$ it follows from that last equality that

$$
P^{*} u=-\Delta u-\left(L^{*}\right)^{2} u=-\left(L^{*}\right)^{2} u=-g \in C^{\infty}\left(\mathbb{T}^{n+m}\right),
$$

which implies that $P^{*}$ is not globally hypoelliptic in $\mathbb{T}^{n+m}$.

Sufficiency: Since $L^{*}$ is globally hypoelliptic in $\mathbb{T}^{m}$ it follows from [CC that there exists a diffeomorphism in $\mathbb{T}^{m}, y=\tau(x)$, such that $L=\sum_{j=1}^{m} a_{j}(x) \partial_{x_{j}}$ can be written as

$$
L_{y}=\sum_{j=1}^{m} A_{j} \partial_{y_{j}}
$$

where the real constants $A_{j}$ satisfy the following Diophantine condition: there exist $K>0, C>0$ such that

$$
\left|\sum_{j=1}^{m} k_{j} A_{j}\right| \geq \frac{C}{(1+|k|)^{K}}, \quad \forall k=\left(k_{1}, \ldots, k_{m}\right) \in \mathbb{Z}^{m} \backslash\{0\} .
$$

In these new variables we can write

$$
P^{*}=-\Delta-\left(L_{y}+b(y)\right)^{2}=-\Delta-Q^{2},
$$

where we are setting

$$
Q=L_{y}+b(y)
$$

with $b \in C^{\infty}\left(\mathbb{T}^{m} ; \mathbb{R}\right)$.

We shall show that $P^{*}$ is globally hypoelliptic in $\mathbb{T}^{n+m}$. For this, let $u \in$ $D^{\prime}\left(\mathbb{T}^{n+m}\right)$ be such that $P^{*} u=f \in C^{\infty}\left(\mathbb{T}^{n+m}\right)$.

Since $L_{y}$ is globally $C^{\infty}$ solvable in $\mathbb{T}^{m}$ there exists $w(y) \in C^{\infty}\left(\mathbb{T}^{m}\right)$ such that

$$
L_{y} w=-b(y)+b_{00},
$$

where $b_{00}=\frac{1}{(2 \pi)^{m}} \int_{\mathbb{T}^{m}} b(y) d y$.

We now set $v=e^{-w} u$. Thus,

$$
L_{y} u=\left(L_{y} w\right) e^{w} v+e^{w} L_{y} v=\left(-b(y)+b_{00}\right) e^{w} v+e^{w} L_{y} v
$$


and therefore we have

$$
\begin{aligned}
Q u & =L_{y} u+b(y) u=\left(-b(y)+b_{00}\right) e^{w} v+e^{w} L_{y} v+b(y) e^{w} v \\
& =b_{00} e^{w} v+e^{w} L_{y} v=e^{w}\left(L_{y} v+b_{00} v\right)
\end{aligned}
$$

and

$$
\begin{aligned}
Q^{2} u & =\left(L_{y}+b(y)\right) e^{w}\left(L_{y} v+b_{00} v\right) \\
& =\left(L_{y} w\right) e^{w}\left(L_{y} v+b_{00} v\right)+e^{w} L_{y}\left(L_{y} v+b_{00} v\right)+b(y) e^{w}\left(L_{y} v+b_{00} v\right) \\
& =b_{00} e^{w}\left(L_{y} v+b_{00} v\right)+e^{w} L_{y}\left(L_{y} v+b_{00} v\right) \\
& =e^{w}\left[\left(L_{y}+b_{00}\right)\left(L_{y} v+b_{00} v\right)\right]=e^{w}\left(L_{y}+b_{00}\right)^{2} v
\end{aligned}
$$

i.e., we have

$$
Q^{2} u=e^{w}\left(L_{y}+b_{00}\right)^{2} v
$$

Thus, we can write

$$
\begin{aligned}
f & =P^{*} u=-\Delta_{t} u-e^{w}\left(L_{y}+b_{00}\right)^{2} v \\
& =e^{w}\left[-\Delta_{t} v-\left(L_{y}+b_{00}\right)^{2} v\right]
\end{aligned}
$$

and therefore we have

$$
-\Delta_{t} v-\left(L_{y}+b_{00}\right)^{2} v=e^{-w} f \doteq g \in C^{\infty}\left(\mathbb{T}^{n+m}\right) .
$$

We now will analyze the operator with constant coefficients:

$$
R=-\Delta_{t}-\left(L_{y}+b_{00}\right)^{2}=-\sum_{j=1}^{n} \partial_{t_{j}}^{2}-\left(\sum_{k=1}^{m} A_{j} \partial_{y_{j}}+b_{00}\right)^{2} .
$$

By taking the Fourier transform in the equation $R v=g$ we obtain

$$
\left[|\tau|^{2}+\left(\sum_{k=1}^{m} A_{j} \xi_{j}-i b_{00}\right)^{2}\right] \hat{v}(\tau, \xi)=\hat{g}(\tau, \xi)
$$

For $\tau=0$ we have

$$
\hat{v}(0, \xi)=\frac{\hat{g}(0, \xi)}{\left(\sum_{k=1}^{m} A_{j} \xi_{j}-i b_{00}\right)^{2}} .
$$

From now on, we will use $C_{N}$ to represent a constant that depends on $N$, which may change a finite number of times.

The fact that $g \in C^{\infty}\left(\mathbb{T}^{3}\right)$ implies that given $N \in \mathbb{N}$ there exists $C_{N}>0$ such that

$$
|\hat{g}(\tau, \xi)| \leq C_{N}(1+|(\tau, \xi)|)^{-N}, \forall(\tau, \xi) \in \mathbb{Z}^{n+m} .
$$

It follows from this, from (4.2) and from (4.4) that given $N \in \mathbb{N}$ there exists $C_{N}>0$ such that

$$
|\hat{v}(0, \xi)| \leq C_{N}(1+|\xi|)^{-N}, \forall \xi \in \mathbb{Z}^{m} .
$$

We now assume that $\tau \neq 0$ and we split this case into two subcases: i) $b_{00}=0$ : given $N \in \mathbb{N}$ there exists $C_{N}>0$ such that

$$
|\hat{v}(\tau, \xi)|=\left|\frac{\hat{g}(\tau, \xi)}{|\tau|^{2}+\left(\sum_{k=1}^{m} A_{j} \xi_{j}\right)^{2}}\right| \leq|\hat{g}(\tau, \xi)| \leq(1+|(\tau, \xi)|)^{-N},
$$

for $\tau \in \mathbb{Z}^{n} \backslash\{0\}$ and $\xi \in \mathbb{Z}^{m}$. 
ii-1) $b_{00} \neq 0$ and $|\tau|^{2} \geq b_{00}^{2}+1$ : since

$$
\left.|| \tau\right|^{2}+\left.\left(\sum_{k=1}^{m} A_{j} \xi_{j}-i b_{00}\right)^{2}|\geq| \tau\right|^{2}+\left(\sum_{k=1}^{m} A_{j} \xi_{j}\right)^{2}-b_{00}^{2} \geq|\tau|^{2}-b_{00}^{2} \geq 1
$$

for $(\tau, \xi) \in \mathbb{Z}^{n+m}$ and $|\tau|^{2} \geq b_{00}^{2}+1$, the estimate for $|\hat{v}(\tau, \xi)|$ follows as in case $\left.\mathbf{i}\right)$. ii-2) $b_{00} \neq 0$ and $\tau \neq 0,|\tau|^{2}<b_{00}^{2}+1$ : in this case we note that

$$
\left.|| \tau\right|^{2}+\left(\sum_{k=1}^{m} A_{j} \xi_{j}-i b_{00}\right)^{2}|\geq 2| b_{00}|| \sum_{k=1}^{m} A_{j} \xi_{j} \mid
$$

and therefore it follows from (4.2) that there exist positive constants $C$ and $K$ such that

$$
\left.|| \tau\right|^{2}+\left(\sum_{k=1}^{m} A_{j} \xi_{j}-i b_{00}\right)^{2}|\geq 2 C| b_{00} \mid(1+|\xi|)^{-K}, \forall \xi \neq 0 .
$$

Summing up, we have proved that given $N \in \mathbb{N}$ there exists $C_{N}>0$ such that

$$
|\hat{v}(\tau, \xi)| \leq C_{N}(1+|(\tau, \xi)|)^{-N}
$$

for $(\tau, \xi) \in \mathbb{Z}^{n+m} \backslash\{F\}$, where $F$ is a finite subset, in turn implies that $v \in$ $C^{\infty}\left(\mathbb{T}^{n+m}\right)$ and therefore $P^{*}$ is globally hypoelliptic in $\mathbb{T}^{n+m}$.

Proof of 2). Necessity: We assume that $P$ is globally hypoelliptic in $\mathbb{T}^{n+m}$ and let $u \in \mathcal{D}^{\prime}\left(\mathbb{T}^{m}\right)$ be such that $L u=f \in C^{\infty}\left(\mathbb{T}^{m}\right)$. Then $P u=-L^{2} u=-L f \in$ $C^{\infty}\left(\mathbb{T}^{m}\right)$. Hence, $u \in C^{\infty}\left(\mathbb{T}^{m}\right)$. The proof of the necessity is complete.

Sufficiency: Since $L$ is globally hypoelliptic in $\mathbb{T}^{m}$ and we are assuming that Ker $L^{*}=[w]$ with $w$ as in Theorem 3.1, it follows from Theorem 3.1 that there exist new variables, $y$, such that the vector field $L$ can be written as

$$
L=\sum_{j=1}^{m} A_{j} \partial_{y_{j}}
$$

where the real numbers $A_{j}$ satisfy the Diophantine condition (3.1). Thus, we can write $P$ as

$$
P=-\Delta-\left(\sum_{j=1}^{m} A_{j} \partial_{y_{j}}\right)^{2}
$$

and therefore, $P$ is globally hypoelliptic in $\mathbb{T}^{n+m}$. This completes the proof of item 2) of Theorem 4.1.

We shall need the following.

Lemma 4.2. Let $P$ be given by (4.1). If $P$ is globally $C^{\infty}$ solvable in $\mathbb{T}^{n+m}$, then $L^{2}$ is globally $C^{\infty}$ solvable in $\mathbb{T}^{m}$.

Proof. Suppose that $L^{2}$ is not globally $C^{\infty}$ solvable. Then there exists $f \in \mathcal{E}\left(L^{2}\right)=$ $\left\{h \in C^{\infty}\left(\mathbb{T}^{m}\right):\langle h, w\rangle=0, \forall w \in \operatorname{Ker}\left(L^{2}\right)^{*}\right\}$ such that $L^{2} u \neq f, \forall u \in C^{\infty}\left(\mathbb{T}^{m}\right)$.

We now define

$$
g(t, x)=\sum_{(\tau, \xi) \in \mathbb{Z}^{n+m}} \hat{g}(\tau, \xi) e^{i(t \cdot \tau+x \cdot \xi)},
$$

where

$$
\hat{g}(\tau, \xi)=\left\{\begin{array}{l}
\hat{f}(\xi) \text { if } \tau=0, \\
0, \quad \text { otherwise }
\end{array}\right.
$$


We now are going to prove that $g \in \mathcal{E}(P)$.

Since $f \in C^{\infty}\left(\mathbb{T}^{m}\right)$ we have $g \in C^{\infty}\left(\mathbb{T}^{n+m}\right)$. Let $w \in \operatorname{Ker} P^{*}$. Then, $P^{*} w=0$ and it is equivalent to $-\Delta w-\left(L^{*}\right)^{2} w=0$. By using a $t$-partial Fourier series, the last equation is equivalent to

$$
|\tau|^{2} \hat{w}(\tau, x)-\left(L^{*}\right)^{2} \hat{w}(\tau, x)=0, \tau \in \mathbb{Z}^{n}, x \in \mathbb{T}^{m} .
$$

In particular, for $\tau=0$ we obtain from the last equality that

$$
\left(L^{*}\right)^{2} \hat{w}(0, x)=0, x \in \mathbb{T}^{m} ;
$$

i.e., $\hat{w}(0, x) \in \operatorname{Ker}\left(L^{*}\right)^{2}$. Therefore, $\hat{w}(0, x) \in \operatorname{Ker}\left(L^{2}\right)^{*}$ since $\left(L^{2}\right)^{*}=\left(L^{*}\right)^{2}$.

Since $f \in \mathcal{E}\left(L^{2}\right)$ and $\hat{w}(0, x) \in \operatorname{Ker}\left(L^{2}\right)^{*}$ we have

$$
\langle f, \hat{w}(0, x)\rangle=0 \text {. }
$$

We now recall that for $T \in D^{\prime}\left(\mathbb{T}^{n+m}\right)$ and $\varphi \in C^{\infty}\left(\mathbb{T}^{n+m}\right)$ the following formula holds:

$$
\langle T, \varphi\rangle=(2 \pi)^{n} \sum_{\tau \in \mathbb{Z}^{n}}\langle\hat{T}(-\tau, x), \hat{\varphi}(\tau, x)\rangle .
$$

Applying this formula with $T=w$ and $\varphi=g$ and taking the definition of $g$ into account we obtain

$$
\langle w, g\rangle=(2 \pi)^{n}\langle\hat{w}(0, x), f(x)\rangle .
$$

It follows from (4.7) and (4.8) that $\langle w, g\rangle=0$, and therefore we have proved that $g \in \mathcal{E}(P)$.

Since by hypothesis $P$ is globally $C^{\infty}$ solvable, there exists $u \in C^{\infty}\left(\mathbb{T}^{n+m}\right)$ such that $P u=g$. The equation $P u=g$ is equivalent to

$$
\left(|\tau|^{2}+L^{2}\right) \hat{u}(\tau, x)=\hat{g}(\tau, x), \tau \in \mathbb{Z}^{n}, x \in \mathbb{T}^{m} .
$$

In particular it follows from the last equality that

$$
L^{2} \hat{u}(0, x)=\hat{g}(0, x)=f(x),
$$

which is a contradiction of the fact that we are assuming that $L^{2}$ is not globally $C^{\infty}$ solvable.

We are now ready to prove the following result:

Theorem 4.3. We assume that $\operatorname{Ker} L^{*}=\operatorname{Ker}\left(L^{*}\right)^{2}=[w]$, where $w \in C^{\infty}\left(\mathbb{T}^{m}\right)$ and $w(x) \neq 0, \forall x \in \mathbb{T}^{m}$. Then $P$ is globally $C^{\infty}$ solvable in $\mathbb{T}^{n+m}$ if and only if $L$ is globally $C^{\infty}$ solvable in $\mathbb{T}^{m}$.

Proof. Necessity: Assuming that $P$ is globally $C^{\infty}$ solvable in $\mathbb{T}^{n+m}$ it follows from Lemma 4.2 that $L^{2}$ is globally $C^{\infty}$ solvable in $\mathbb{T}^{m}$. We will show that $L$ is globally $C^{\infty}$ solvable in $\mathbb{T}^{m}$. For this, let $f \in \mathcal{E}(L)$. Thanks to the fact that $\operatorname{Ker} L^{*}=\operatorname{Ker}\left(L^{*}\right)^{2}$ we can conclude that $f \in \mathcal{E}\left(L^{2}\right)$. Since $L^{2}$ is globally $C^{\infty}$ solvable in $\mathbb{T}^{2}$ and $f \in \mathcal{E}\left(L^{2}\right)$ there exists $u \in C^{\infty}\left(\mathbb{T}^{m}\right)$ such that $L^{2} u=f$ and therefore $v=L u \in C^{\infty}\left(\mathbb{T}^{m}\right)$ satisfies the equation

$$
L v=f ;
$$

i.e., $L$ is globally $C^{\infty}$ solvable in $\mathbb{T}^{m}$.

Sufficiency: Since, by hypothesis, $L$ is globally $C^{\infty}$ solvable in $\mathbb{T}^{m}$ and $\operatorname{Ker} L^{*}=$ $[w]$ where $w \in C^{\infty}\left(\mathbb{T}^{m}\right)$ and $w(x) \neq 0, \forall x \in \mathbb{T}^{m}$, it follows from Theorem 3.1 that $L^{*}$ is globally hypoelliptic in $\mathbb{T}^{m}$. It now follows from Theorem 4.1 that $P^{*}$ is globally hypoellipitic in $\mathbb{T}^{n+m}$ and therefore $P$ is globally solvable in $\mathbb{T}^{n+m}$. But, 
it also follows from Theorem 3.1 and Theorem 4.1 that $P$ is globally hypoelliptic in $\mathbb{T}^{n+m}$ and therefore we now can conclude that $P$ is globally $C^{\infty}$ solvable in $\mathbb{T}^{n+m}$.

We now will present an example of a vector field, $X$, for which we have $\operatorname{Ker} X^{*}=$ $\operatorname{Ker}\left(X^{*}\right)^{2}=[w]$, where $w \in C^{\infty}\left(\mathbb{T}^{m}\right)$ and $w(x) \neq 0, \forall x \in \mathbb{T}^{m}$.

Example 4.4. We consider in $\mathbb{T}^{3}$ the vector field

$$
X=\partial_{t}+a(t) \partial_{x}+b(t) \partial_{y},
$$

where $a, b \in C^{\infty}(\mathbb{T})$ are real-valued. We are assuming that the vector $\left(a_{0}, b_{0}\right)$ is a non-Liouville vector, where

$$
a_{0}=\frac{1}{2 \pi} \int_{0}^{2 \pi} a(t) d t, b_{0}=\frac{1}{2 \pi} \int_{0}^{2 \pi} b(t) d t .
$$

We recall that a vector $v=\left(v_{1}, v_{2}\right) \in \mathbb{R}^{2}$ is said to be non-Liouville if there exist $C>0$ and $K>0$ such that for any $\eta \in \mathbb{Z}$ and $\xi \in \mathbb{Z}^{2} \backslash\{0\}$ we have

$$
|\eta-v \cdot \xi| \geq \frac{C}{|\xi|^{K}} .
$$

We shall need the following.

Lemma 4.5. Under the above conditions the vector field $X$ is globally hypoelliptic in $\mathbb{T}^{3}$.

Proof. Let $u \in D^{\prime}\left(\mathbb{T}^{3}\right)$ be such that

$$
X u=f \in C^{\infty}\left(\mathbb{T}^{3}\right) .
$$

By taking the Fourier transform with respect to the variables $x$ and $y$ in (4.9) we obtain

$$
\partial_{t} \hat{u}(t, \xi, \eta)+i \xi a(t) \hat{u}(t, \xi, \eta)+i \eta b(t) \hat{u}(t, \xi, \eta)=\hat{f}(t, \xi, \eta) .
$$

For each $(\xi, \eta) \in \mathbb{Z}^{2}$ the operator in equation (4.10) is elliptic in $t$ and therefore by elliptic theory we have $\hat{u}(\cdot, \xi, \eta) \in C^{\infty}(\mathbb{T})$. We set

$$
A(t)=\int_{0}^{t} a(s) d s-a_{0} t, B(t)=\int_{0}^{t} b(s) d s-b_{0} t
$$

and

$$
v(t, \xi, \eta)=e^{i \xi A(t)+i \eta B(t)} \hat{u}(t, \xi, \eta) .
$$

Thus, $v(t, \xi, \eta)$ satisfies the equation

$$
\partial_{t} v(t, \xi, \eta)+i\left(a_{0} \xi+b_{0} \eta\right) v(t, \xi, \eta)=\hat{f}(t, \xi, \eta) e^{i \xi A(t)+i \eta B(t)} \doteq g(t, \xi, \eta) .
$$

For $(\xi, \eta) \in \mathbb{Z}^{2} \backslash\{0\}$ such that $\frac{1}{e^{2 \pi i\left(a_{0} \xi+b_{0} \eta\right)}-1} \neq 0$ we have

$$
v(t, \xi, \eta)=\frac{1}{e^{2 \pi i\left(a_{0} \xi+b_{0} \eta\right)}-1} \int_{0}^{2 \pi} e^{i\left(a_{0} \xi+b_{0} \eta\right) s} g(t+s, \xi, \eta) d s .
$$

Since the vector $\left(a_{0}, b_{0}\right)$ is non-Liouville it is easily seen that there exist $C>0$ and $K>0$ such that

$$
\left|e^{2 \pi i\left(a_{0} \xi+b_{0} \eta\right)}-1\right| \geq \frac{C}{|(\xi, \eta)|^{K}}
$$

for all $(\xi, \eta) \in \mathbb{Z}^{2} \backslash\{0\}$. 
For $\xi=\eta=0$ we have

$$
v(t, 0,0)=\int_{0}^{t} g(s, 0,0) d s+C
$$

with $\int_{0}^{2 \pi} g(s, 0,0) d s=0$ and $C$ is a constant.

It is standard to prove that (4.12), (4.13) and (4.14) imply that $X$ is globally hypoelliptic in $\mathbb{T}^{3}$.

Remark 4.6. In particular, (4.12), (4.13) and (4.14) imply that

$$
\operatorname{Ker} X=[1] \text {. }
$$

We now notice that

$$
X^{*}=-X \text { and }\left(X^{*}\right)^{2}=X^{2} .
$$

Suppose that $X^{2} u=0$. Then, $X(X u)=0$ and therefore it follows from (4.15) that there exists a constant $C$ such that $X u=C$. Thus, $C$ must satisfy the compatibility condition

$$
(2 \pi)^{3} C=\langle C, 1\rangle=\langle X u, 1\rangle=\left\langle u, X^{*} 1\right\rangle=\langle u,-X 1\rangle=\langle u, 0\rangle=0 .
$$

Hence, $C=0$ and we have proved that

$$
\operatorname{Ker} X^{2} \subset \operatorname{Ker} X \text {. }
$$

Summing up we have proved that

$$
\operatorname{Ker} X^{*}=\operatorname{Ker}\left(X^{*}\right)^{2} \text {. }
$$

It follows from Theorem 3.1 that the vector field $X$ is globally $C^{\infty}$ solvable in $\mathbb{T}^{3}$, and it follows from Theorem 4.3 that the operator $P=-\Delta-X^{2}$ is globally $C^{\infty}$ solvable in $\mathbb{T}^{n+3}$.

Remark 4.7. Since $X$ is globally $C^{\infty}$ solvable in $\mathbb{T}^{3}$, then let $\varphi_{1}, \varphi_{2}, \varphi_{3} \in C^{\infty}\left(\mathbb{T}^{3}\right)$ be such that

$$
X \varphi_{1}=0, X \varphi_{2}=a_{0}-a(t), X \varphi_{3}=b_{0}-b(t) .
$$

For this example it is easy to see that the diffeomorphism $\tau=\tau(t, x, y)$, which is guaranteed by Theorem 3.1, is given by

$$
\begin{gathered}
\tau_{1}(t, x, y)=t+\varphi_{1}(t, x, y), \\
\tau_{2}(t, x, y)=x+\varphi_{2}(t, x, y), \\
\tau_{3}(t, x, y)=y+\varphi_{3}(t, x, y)
\end{gathered}
$$

and the constants $A_{1}, A_{2}, A_{3}$ are given by

$$
A_{1}=1, A_{2}=a_{0}, A_{3}=b_{0} .
$$

It is also easily seen that the condition that $\left(a_{0}, b_{0}\right)$ is a non-Liouville vector implies the Diophantine condition (3.1). 


\section{REFERENCES}

[AZ] A. A. Albanese and L. Zanghirati, Global hypoellipticity and global solvability in Gevrey classes on the n-dimensional torus, J. Differential Equations 199, (2004), 256-268. MR2047910(2005a:35049)

[A] K. Amano, The global hypoellipticity of a class of degenerate elliptic-parabolic operators, Proc. Japan Acad. 60, Ser. 4, (1984), 312-314. MR778515 (87f:35054)

[BCP] A. P. Bergamasco, P. D. Cordaro and G. Petronilho, Global solvability for a class of complex vector fields on the two-torus, Comm. in PDE 29, no. 5 and 6, (2004), 785-819. MR.2059148 (2005k:35034)

[Br] A. D. Brjuno, Analytic form of differential equations, Trans. Moscow Math. Soc. 25, (1971), 131-288 and 26, (1972), 199-239. MR0377192 (51:13365)

[CC] W. Chen and M.Y. Chi, Hypoelliptic vector fields and almost periodic motions on the torus $T^{n}$, Comm. in PDE 25(1-2), (2000), 337-354. MR.1737551 (2000m:35042)

[FO] D. Fujiwara and H. Omori, An example of a globally hypoelliptic operator, Hokkaido Math. J. 12, (1983), 293-297. MR719969 (86a:35038)

[GPY1] T. Gramchev, P. Popivanov and M. Yoshino, Global solvability and hypoellipticity on the torus for a class of differential operators with variable coefficients, Proc. Japan Acad., 68, (1992), 53-57. MR1167986 (93g:35025)

[GPY2] T. Gramchev, P. Popivanov and M. Yoshino, Global properties in spaces of generalized functions on the torus for second order differential operators with variable coefficients, Rend. Sem. Mat. Pol. Torino 51, no.2, (1993), 145-172. MR.1289385 (95k:35047)

[GW1] S. J. Greenfield and N. R. Wallach, Global hypoellipticity and Liouville numbers, Proc. Amer. Math. Soc., 31 (1972), 112-114. MR0296508 (45:5568)

[GW2] S. J. Greenfield and N. R. Wallach, Globally hypoelliptic vector field, Topology 12, (1973), 247-253. MR0320502 (47:9039)

[He] M. R. Herman, Sur la conjugaison différentiable des difféomorphismes du cercle à des rotations, Publ. I.H.E.S. 49, (1979), 5-233. MR.538680(81h:58039)

[Hi] A. A. Himonas, On degenerate elliptic operators of infinite type, Math. Z. 220, (1995), 449-460. MR 1362255 (96j:35089)

[HP1] A. A. Himonas and G. Petronilho, Global hypoellipticity and simultaneous approximability, J. Funct. Anal., 170, (2000), 356-365. MR.1740656 (2000m:35043)

[HP2] A. A. Himonas and G. Petronilho, On $C^{\infty}$ and Gevrey regularity of sublaplacians, Trans. Amer. Math. Soc. 358, (2006), no. 11, 4809-4820. MR2231873 (2007b:35057)

[HPS] A. A. Himonas, G. Petronilho and L. A. C. dos Santos, Analytic, Gevrey and $C^{\infty}$ global regularity for a class of subLaplacinas, pre-printed.

[Hou] J. Hounie, Globally hypoelliptic vector field on compact surfaces, Comm. in PDE 7(4), (1982), 343-370. MR652813 (83k:35025)

[OK] H. Omori and T. Kobayashi, Global hypoellipticity of subelliptic operators on closed manifolds, Hokkaido Math. J., 28, (1999), 613-633. MR1723457 (2000k:35054)

[P1] G. Petronilho, Global solvability and simultaneously approximable vectors, J. Differential Equations, 184, (2002), 48-61. MR.1929145 (2004g:35047)

[P2] G. Petronilho, On Gevrey solvability and regularity, Math. Nachr., 282, no. 3, (2009), 470-481. MR 2503164 (2010d:35007)

[Y] J.C. Yoccoz, Conjugaison différentiable des difféomorphismes du cercle dont le nombre de rotation vérifie une condition Diophantienne, Ann. Sci. École Norm. Sup. IV Sér. 17, (1984), 333-359. MR777374 (86j:58086)

Departamento de Matemática, Universidade Federal de São Carlos, São Carlos, SP 13565-905, BRAZIL

E-mail address: gersonpetro@gmail.com 